\title{
Editorial to the special issue on Particle background for space instrumentation in the context of the Athena mission
}

\author{
Lorenzo Natalucci $^{1} \cdot$ Claudio Macculi $^{1}$
}

Published online: 20 December 2017

(C) Springer Science+Business Media B.V., part of Springer Nature 2017

In the framework of integrating activities for high energy astrophysics, AHEAD (funded by the EU Horizon 2020 Research Infrastructure Programme) has among its main goals to boost the efforts of the European community for the scientific exploitation of current high energy missions and the design of future space observatories. To this aim AHEAD is fostering modelling studies to support the design of future X-ray and gamma-ray missions, with special regard to Athena.

Athena is the future Large X-ray observatory under development by the European Space Agency with the support of a large community of astronomers. To support at best the Athena instrument design and performance, AHEAD has undertaken initiatives to advance our understanding of the instrumental background in orbit. These studies involve space environment and radiation effects, characterization of primary and secondary background components, Geant 4 simulations, modelling of proton reflection in optics devices, ray tracing, planning of experimental tests.

This special issue of Experimental Astronomy, fostered by AHEAD is a great opportunity to put together the results of the specialised efforts of the experimental teams worlwide and obtain a detailed picture of the current status of knowledge in the field. We wish to thank the authors, the reviewers and the Experimental Astronomy staff for their assistance in the publication of the volume.

Lorenzo Natalucci

Lorenzo.Natalucci@iaps.inaf.it

1 INAF-Istituto di Astrofisica e Planetologia Spaziali, Via del Fosso del Cavaliere, 100, 00133 Rome, Italy 